\title{
FACTORES ASOCIADOS AL PERFIL DE LÍPIDOS SANGUIINEOS EN LOS PACIENTES DEL ÁREA DE SALUD DE MONTES DE OCA, COSTA RICA
}

\author{
Erick G. Gutiérrez Peña y Juan José Romero Zúñiga ${ }^{2}$ \\ ${ }^{1}$ Programa de Atención Integral de Salud, Universidad de Costa Rica \\ ${ }^{2}$ Postgrado Regional en Ciencias Veterinarias Tropicales, Universidad Nacional \\ jromero@medvet.una.ac.cr
}

RESUMEN

En este estudio se analiza la asociación entre la exposición a diversos factores — de biotipo, socioeconómicos y patológicos- y la alteración del perfil de lípidos sanguíneos (dislipidemia) en pacientes adscritos al Área de Salud de Montes de Oca. Se realizó un estudio de caso-control, con un total de 135 casos e igual cantidad de controles, entre 20 y 65 años, a los que se les hizo un perfil de lípidos sanguíneos durante el año 2006. Las variables estudiadas fueron: edad, sexo, índice de masa corporal, tipo de aseguramiento, estado de portador de hipertensión arterial, de diabetes mellitus o de ambas patologías crónicas a la vez. Se realizó un análisis univariado, seguido de un análisis multivariado, mediante un modelo logístico múltiple. La única variable asociada con la dislipidemia fue el índice de masa corporal, tanto en el análisis univariado como en el multivariado; las variables restantes no mostraron asociación estadística. Aquellos pacientes con mayor índice de masa corporal presentan un mayor riesgo de tener un perfil alterado de lípidos sanguíneos.

PALABRAS CLAVE: colesterol, triglicéridos, dislipidemia, factores asociados, Costa Rica.

FACTORS ASSOCIATED WITH THE BLOOD LIPID PROFILE OF PATIENTS IN THE MONTES DE OCA HEALTH SERVICE AREA IN COSTA RICA

\section{ABSTRACT}

This study is aimed at analyzing the association between alterations in the blood lipid profile (dyslipidemia) and the exposure to socioeconomic, biotype and pathological conditions in patients in the Montes de Oca Health Service Area. A case-control study was conducted with a total of 135 cases and 135 controls that were between 20 and 65 years of age and were tested in 2006 for a blood lipid profile. Variables analyzed were: age, sex, body mass index, insurance type and presence of arterial hypertension, diabetes mellitus or both chronic pathologies simultaneously. A univariate analysis was followed by a multivariate analysis using a multiple logistic model. The only variable associated with dyslipidemia was body mass index in both the univariate and multivariate analyses. The rest of the variables showed no statistical association. Patients with higher body mass index exhibit a higher risk of an altered blood lipid profile.

KEYWORDS: cholesterol, triglycerides, dyslipidemia, associated factors, Costa Rica.

$\begin{array}{ll}\text { ABREVIATURAS } & \\ & \\ \text { Área de Salud de Montes de Oca } & \text { ASMO } \\ \text { Enfermedades Cardiovasculares } & \text { ECV } \\ \text { Índice de Masa Corporal } & \text { IMC } \\ \text { Diabetes mellitus } & \text { DM } \\ \text { Odds Ratio } & \text { OR }\end{array}$




\section{INTRODUCCIÓN}

Las enfermedades cardiovasculares (ECV) constituyen la principal causa de mortalidad en el país, con tasas que superan por amplio margen a cualquiera de las otras patologías que afectan a la población (OPS, 2005). Varios estudios han demostrado que, a medida que los pacientes alcanzan un perfil de lípidos sanguíneo (PLS) con valores óptimos, el riesgo de sufrir ECV se disminuye (Law et al., 1994; Collins et al., 2002; Shepherd et al., 2002). El control del PLS es, entonces, uno de los pilares en la prevención primaria de estas patologías.

La presencia de niveles anormales de lípidos sanguíneos puede estar determinada por diversos factores. La predisposición genética es uno de ellos (Kathiresan et al., 2007), pero hacer estudios de este tipo resulta muy costoso y no es algo que esté al alcance de la mayoría de la población. Otro factor que hay que tomar en consideración es el sexo, ya que se han reportado variaciones en las concentraciones de todos los lípidos sanguíneos relacionadas con el sexo, que se traducen en indicadores, como el riesgo, la prevalencia, las hospitalizaciones, la morbilidad y la mortalidad, asociados a ECV, distintos para hombres y mujeres" (Ueshima et al., 2006; Jafar et al., 2007; Pilote et al., 2007). Un tercer factor, muy estudiado, es la obesidad. En este campo, se ha observado que al producirse un aumento en el peso se activan una serie de eventos fisiológicos que concluyen con el desarrollo de patologías metabólicas y con la aparición de complicaciones cardiovasculares (Bakris, 2007).

El riesgo de padecer de ECV aumenta en pacientes portadores de patologías crónicas, ya que se produce un efecto sinérgico entre la patología de fondo y los PLS alterados. Los pacientes con diabetes mellitus presentan un incremento en la frecuencia de ECV, por lo que el control de los lípidos sanguíneos es un pilar fundamental en la prevención y el control de la patología cardiovascular en pacientes con diabetes (Watkins, 2003; Betteridge, 2005). De igual forma sucede cuando se presenta HTA, pues se ha clasificado al PLS anormal como un agravante en el riesgo de desarrollar ECV; de ahí que el tratamiento que se les da a estos pacientes busca lograr que sus niveles de lípidos sean los más óptimos posibles (Grundy et al, 2002).

Esta investigación se realizó en el Área de Salud Montes de Oca (ASMO), que pertenece al Programa de Atención Integral en Salud (PAIS) de la Universidad de Costa Rica (UCR). Este programa está compuesto por 15 Equipos Básicos de Atención Integral en Salud (EBAIS), distribuidos en cinco sedes, donde se brindan servicios de primer nivel de atención a una población con características socioeconómicas y culturales variadas.

Se piensa que una gran cantidad de personas sufre de alteraciones en el PLS, lo que constituye un problema de salud local; sin embargo, se desconoce cuáles son los factores que están asociados a este fenómeno y en qué medida influyen en él. El objetivo de este estudio es analizar la relación entre la exposición a diversos factores — de biotipo, socioeconómica y patológica- y la manifestación de alteraciones en el PLS en pacientes del ASMO.

\section{METODOLOGIA}

Se realizó un estudio de tipo caso-control (Rottman, 1986). La población objetivo estuvo compuesta por aproximadamente 38000 pacientes de entre 20 y 65 años de edad, adscritos al 


\section{MHSalud \\ URL $\underline{\text { www.una.ac.cr/mhsalud }}$}

ASMO. El diseño del estudio fue aprobado por un comité evaluador del Posgrado en Epidemiología de la Universidad Nacional, designado para evaluar los aspectos técnicos y éticocientíficos. Además, se contó con la aprobación de la Coordinación Académica y la Dirección General del PAIS, que es la entidad responsable de avalar la ejecución de investigaciones y de la divulgación de información en este programa.

\section{Sujetos}

La información de la población participante se recopiló entre el $1^{\circ}$ de enero y el 31 de diciembre del 2006, consultando el registro del Laboratorio Clínico de la Universidad de Costa Rica. Se trabajó con los expedientes de los pacientes a los que se les había practicado un PLS durante el período de estudio. Formaron parte del estudio los pacientes que cumplían con los siguientes requisitos: adscripción a un EBAIS perteneciente al ASMO, indicación médica del PLS, como parte de su control de salud, y edad entre 20 y menos de 65 años. Se excluyeron de esta población los pacientes cuyos expedientes presentaban al menos una de las siguientes carencias: reportes biológicamente no plausibles; falta de datos, o datos mal digitados o en blanco; valores mayores a 3 desviaciones estándares, o reportes diferentes al primer PLS indicado en el año. En estos casos, se conservó el primer reporte y se eliminaron los restantes. El protocolo de los EBAIS establece que para determinar un perfil metabólico únicamente se tomen muestras sanguíneas a pacientes que tengan al menos 12 horas de ayuno.

Se definieron como "caso" aquellas personas que presentaban el PLS alterado, o sea, que cumplían con al menos uno de los siguientes criterios: colesterol total $\geq 240 \mathrm{mg} / \mathrm{dl}$, triglicéridos $\geq 200 \mathrm{mg} / \mathrm{dl}$, LDL-colesterol $\geq 160 \mathrm{mg} / \mathrm{dl}$ o HDL-colesterol $<40 \mathrm{mg} / \mathrm{dl}$. Se definieron como "control" aquellas personas que presentaban un PLS óptimo, en apego a los siguientes criterios: colesterol total <240 mg/dl, triglicéridos <200 mg/dl, LDL-colesterol <160 mg/dl, HDLcolesterol $\geq 40 \mathrm{mg} / \mathrm{dl}$ (Adult Treatment Panel III, 2001)

Se utilizaron métodos estandarizados y validados para la determinación de los lípidos sanguíneos y la glucosa sanguínea. Para la toma de la presión arterial se emplearon esfigmomanómetros de tipo aneroide.

El tamaño de la muestra se determinó según la fórmula $\mathrm{n}=\Sigma(\mathrm{k}-1 * 10)$, donde $\mathrm{k}$ es el número de estratos de las variables; por lo que toma en cuenta todos los niveles de cada covariable. Esta fórmula es una variación de la fórmula de Freeman (1987) $n=10^{*}(k+1)$, donde $k$ es el número total de covariables. Esta corrección permite tener al menos las 10 observaciones por covariable que sugiere Freeman, pero incrementa el total de observaciones cuando las covariables tienen más de dos estratos, acercándose a lo señalado por Harrel et al., (1985) y Perduzzi et al. (1996), que recomiendan entre 10 y 20 observaciones por variable, así como el número de observaciones derivadas de las posibles interacciones. El resultado fue de 100 pacientes por grupo; sin embargo, dada la posibilidad de obtener información de un mayor número de pacientes, se trabajó con 135 casos y 135 controles.

La distribución del número de pacientes fue proporcional al aporte que cada una de las cinco sedes del ASMO realizó a la población total durante al período de estudio. La selección de los expedientes en cada una de las sedes se hizo de forma aleatoria simple entre el total de pacientes registrados. Cuando los datos estaban incompletos o faltaban del todo (ausentes), se acudió al reemplazo simple. Para ambos grupos ("casos" y "controles") se seleccionaron igual cantidad de pacientes hombres que de pacientes mujeres. 
URL www.una.ac.cr/mhsalud

\section{Variables de estudio}

Las variables o factores de exposición que se incluyeron en el estudio fueron:

Índice de Masa Corporal (IMC): resultado de dividir el peso en kilogramos entre el cuadrado de la estatura en metros $\left[\mathrm{kg} /(\mathrm{m})^{2}\right]$. Se definieron tres categorías para esta variable: normal, para valores entre 20,1 a 25,0; sobrepeso, para valores de 25,1 a 30,0 y obesos, para valores $>30,0$. Si bien la Organización Mundial de la Salud (1995) establece como normal un IMC de 18,5 a 24,99, en nuestra investigación utilizamos el rango de 20,1 a 25,0 como IMC normal, debido al uso que ordinariamente se le confiere a este valor en el ámbito de los profesionales en medicina, nutrición y ciencias del movimiento humano (Koloszár et al., 2005; Hallin et al., 2006; Mantakas \& Farrel, 2010; Renault et al. 2010).

Hipertensión arterial (HTA) y diabetes mellitus (DM) tipo II: indica si un paciente es portador o no de estas patologías. Se trabajó cada variable de forma independiente (HTA o DM) pero también se creó una variable que las contemplaba como co-morbilidades (HTA/DM). Para definir la hipertensión arterial se utilizó el valor de 140/90 $\mathrm{mmHg}$ (sistólica/diastólica), según las recomendaciones del Seventh Report of the Joint National Committee on Prevention, Detection, Evaluation, and Treatment of High Blood Pressure (US Department of Health and Human Services (2004)). Por otro lado, se utilizó el valor de $125 \mathrm{mg} / \mathrm{dl}$ como valor límite normal de glucosa en sangre (suero venoso) en ayunas (Resnick et al., 2000).

Tipo de aseguramiento: condición de afiliación a la Caja Costarricense de Seguro Social que presenta cada uno de los pacientes, según su estado socioeconómico. Se definieron tres categorías: contribuyentes (asegurados directos o pensionados directos), dependientes (cónyuge o los hijos de un contribuyente) y otros asegurados (pacientes con seguro del Estado, convenios internacionales o condición no determinada a la fecha).

Edad: se organizó en tres clases; pacientes de 20 a 34 años, de 35 a 49 años y de 50 a menos de 65 años cumplidos.

Sexo: masculino o femenino, según lo registrado en el expediente.

La información de cada variable se encontró en los expedientes clínicos de cada uno de los pacientes y se tabularon los datos correspondientes a la fecha en la que se indicó el PLS durante el 2006.

\section{Análisis estadístico}

En la primera parte del estudio se llevó a cabo un análisis univariado por medio de tablas de contingencia ( $2 \mathrm{x} \mathrm{k})$ para calcular, mediante la razón de posibilidades (Odds Ratio [OR]), la magnitud de la asociación entre el PLS y cada una de las variables de exposición. El intervalo de confianza de cada OR se estimó al 95\% ( $\mathrm{IC}_{95 \%}$ ). Además, se evaluó la dirección, la magnitud y la fuerza de la asociación de cada OR resultante.

En la segunda parte se construyó un modelo multivariado, siguiendo la estrategia de modelaje "paso a paso en reversa" (backward stepwise). La prueba de significancia que se usó durante el modelaje multivariado fue la razón de verosimilitud (Likelihood Ratio Test), con un nivel de confianza del 95\%. Luego de la exclusión de las variables no significativas, se evaluó la presencia de confusión comparando los coeficientes estimados en el nuevo modelo, con los calculados y 


\section{MHSalud}

URL $\underline{\text { www.una.ac.cr/mhsalud }}$

con la razón de verosimilitud del modelo previo. Se consideró la presencia de confusión cuando el coeficiente de alguna de las variables de exposición cambió en más del 10\% (si el coeficiente tenía valores entre $-0,4$ y 0,4 ) o si los coeficientes cambiaban más del $25 \%$ (si el coeficiente tenía valores de $<-0,4$ o >0,4). Finalmente, las interacciones biológicamente plausibles fueron agregadas al modelo y evaluadas estadísticamente.

El modelo multivariado para evaluar los distintos PLS fue el siguiente:

$$
\operatorname{Logit}(P) P L S=\alpha+\beta I M C+\beta H T A+\beta D M+\beta H T A / D M+\beta A+\gamma E d a d+\gamma \operatorname{Sexo}
$$

El software que se empleó para ambos análisis fue Egret ${ }^{\circledR}$ versión 2.0.31 (Cytel Software Corp.)

\section{RESULTADOS}

Del total de pacientes, tanto "casos" como "control", 90 tenían entre 20 y 34 años, 84 estaban entre los 35 y los 49 años y 96 tenían entre 50 y $\leq 65$ años. La distribución por sexo fue similar en ambos grupos (casos y control). Se encontró que había 75 pacientes con un IMC normal, 122 con sobrepeso y 73 con obesidad. Un total de 40 pacientes eran portadores de HTA, 11 presentaron DM y 3, ambas patologías. En relación con la condición de aseguramiento, 156 pacientes fueron categorizados como "contribuyentes", 63 como "dependientes" y 51 como "otros asegurados".

En el análisis univariado se encontró que las variables edad, sexo, aseguramiento, DM y HTA/DM no presentaban una asociación significativa con el PALS. En cambio, el IMC mostró una alta asociación estadística con el PALS, especialmente en los pacientes obesos. La HTA también evidenció algún grado de asociación (Tabla 1).

Tabla 1.

Análisis univariado para los factores asociados con el perfil alterado de lípidos sanguíneos. Área de Salud Montes de Oca, 2006.

\begin{tabular}{llccc}
\hline Variable & \multicolumn{1}{c}{ Nivel } & OR & $\mathrm{IC}_{95 \%}$ & $p$ \\
\hline Edad & $20-34$ & - & - & - \\
& $35-49$ & 1,06 & $0,58-1,90$ & 0,88 \\
Sexo & $50-64$ & 1,09 & $0,61-1,94$ & 0,77 \\
& Masculino & - & & \\
IMC & Femenino & 0,97 & $0,60-1,56$ & 0,90 \\
& $<25$ & - & - & - \\
& $25-30$ & 1,88 & $1,04-3,41$ & 0,03 \\
HTA & $>30$ & 3,62 & $1,84-7,13$ & $<0,00$ \\
DM & Portador & 2,06 & $1,02-4,15$ & 0,04 \\
HTA y DM & Portador & 2,77 & $0,72-10,68$ & 0,14 \\
Aseguramiento & Portador & 2,02 & $0,18-22,49$ & 0,57 \\
& Contribuyente & - & - & - \\
& Dependiente & 1,17 & $0,65-2,11$ & 0,59 \\
& Otros & 1,62 & $0,86-3,08$ & 0,14 \\
\hline
\end{tabular}

En el modelo multivariado, las variables edad, sexo, HTA, DM, HTA/DM y aseguramiento no mostraron significancia estadística; por el contrario, solo el IMC continuó mostrando asociación con el PALS (Tabla 2). 


\section{MHSalud}

URL $\underline{\text { www.una.ac.cr/mhsalud }}$

Una vez eliminadas las variables que no presentaban asociación, el modelo predictivo que resultó con mayor significancia estadística $(p<0,00)$, según la razón de verosimilitud fue:

$$
\text { Logit }(P) P L S=\alpha+\beta I M C
$$

No se presentó confusión o modificación de efecto con las variables esperadas para tales interacciones.

Tabla 2.

Análisis multivariado para los factores asociados al tipo de perfil alterado de lípidos sanguíneos. Área de Salud Montes de Oca, 2006.

\begin{tabular}{llccc}
\hline Variable & \multicolumn{1}{c}{ Nivel } & OR & $\mathrm{IC}_{95 \%}$ & $p$ \\
\hline Edad & $20-34$ & - & - & - \\
& $35-49$ & 0,86 & $0,46-1,63$ & 0,64 \\
Sexo & $50-64$ & 0,67 & $0,37-1,31$ & 0,25 \\
& Masculino & - & & \\
IMC & Femenino & 0,94 & $0,55-1,60$ & 0,82 \\
& $<25$ & - & - & - \\
& $25-30$ & 1,98 & $1,06-3,71$ & 0,03 \\
HTA & $>30$ & 3,36 & $1,65-6,86$ & $<0,00$ \\
DM & Portador & 1,90 & $0,87-4,13$ & 0,10 \\
HTA y DM & Portador & 2,14 & $0,52-8,79$ & 0,29 \\
Aseguramiento & Portador & 1,84 & $0,15-22,96$ & 0,63 \\
& Contribuyente & - & - & - \\
& Dependiente & 1,16 & $0,60-2,22$ & 0,67 \\
& Otros & 1,70 & $0,85-3,40$ & 0,14 \\
\hline
\end{tabular}

\section{DISCUSIÓN}

Los hallazgos de este estudio se basaron en las definiciones de perfil alterado de lípidos sanguíneos (PALS) y de perfil óptimo de lípidos sanguíneos (POLS) establecidas en la metodología. Este aspecto es de suma importancia para la validez externa del estudio y para su aplicación en otras circunstancias La literatura (Grundy et al., 2002) menciona que, en algunos pacientes, se deben considerar como óptimos valores de LDL-colesterol de $<100 \mathrm{mg} / \mathrm{dl}$ y no como se definió en la investigación $(<160 \mathrm{mg} / \mathrm{dl})$; por lo tanto, los resultados de este estudio deben usarse con cautela en esos pacientes.

A pesar de que se han documentado diferencias en la concentración de lípidos sanguíneos relacionadas con el sexo (Tacsan \& Asencio, 2001), en este estudio no se precisó una asociación significativa entre el sexo y el PALS. Esta situación podría deberse a que el PALS se consideró como un todo y no se analizó el efecto de esta variable en cada lípido sanguíneo de forma individual. Por ello, el efecto del sexo en un determinado lípido no fue evidenciado.

En la investigación tampoco se estableció una asociación significativa entre las distintas categorías de edad y el PALS. Esto se debe, posiblemente, a que se crearon clases de edad con intervalos de años relativamente amplios. Este ámbito pudo haber obstaculizado la observación de alguna tendencia, pero, en todo caso, de haber existido se habría notado en las categorías extremas, cosa que no ocurrió. 


\section{MHSalud \\ URL www.una.ac.cr/mhsalud}

El IMC fue la variable que tuvo la asociación estadística de mayor significancia. Se comprobó que el riesgo de tener una alteración en el PLS aumenta en los pacientes con mayor IMC. Este hallazgo encuentra refuerzo en la literatura, que señala una relación directa entre los diferentes estados de obesidad, los lípidos sanguíneos y las ECV (Klein et al., 2004; Meigs et al., 2006; Poirier et al., 2006).

A pesar de que las alteraciones en los diferentes lípidos sanguíneos son comunes en los pacientes portadores de patologías crónicas, el estudio solo demostró asociación significativa con la HTA, no así con la DM o con la presencia de ambas. Una posible justificación para este hallazgo es el hecho de que los pacientes portadores de estas patologías se encuentran cumpliendo los lineamientos de los protocolos respectivos, que establecen el abordaje de las alteraciones en los lípidos sanguíneos y que los convierte en pacientes que están recibiendo tratamiento antilipídico.

No existe documentación disponible que relacione la condición de aseguramiento de una persona con la presentación de un PLS en particular. La plausibilidad biológica de una asociación es nula y esto se confirmó mediante el valor estadístico que se obtuvo en el análisis. A su vez, esta variable no determina la condición socioeconómica de una persona, que está determinada por múltiples factores.

El resultado del análisis multivariado fue un modelo simple (una sola variable), ya que los demás factores de exposición se fueron excluyendo progresivamente por no poseer valores estadísticamente relevantes. En todo caso, la ecuación resultante tiene una alta significancia estadística $(p<0.00)$ como predictor de PALS.

Importa destacar que, si bien el modelo simple demuestra la importante relación entre los factores de exposición y el PALS, otros modelos que incluyan más variables proporcionarían una predicción más explicativa del evento, dada la multi-causalidad de las enfermedades (Kleinbaum, 1994).

\section{CONCLUSIONES}

Una vez analizadas todas las asociaciones de las variables incluidas, se concluye que el IMC fue el único factor que mostró una asociación significativa con el PALS en los pacientes del ASMO. Conociendo esta situación, es pertinente crear estrategias locales para minimizar el riesgo de que se presenten alteraciones en los lípidos sanguíneos y reducir, así, las posibilidades de que se presenten ECV en la población en general, y en la población del ASMO, en particular.

\section{AGRADECIMIENTOS}

A la Dra. Sandra Meléndez, al Prof. Bernardo Vargas, PhD y a la Dra. Gabriela Salguero, por sus aportes en el desarrollo de esta investigación.

\section{REFERENCIAS}

Adult Treatment Panel III. (2001). Expert Panel on Detection, Evaluation, and Treatment of High Blood Cholesterol in Adults. Executive Summary of the Third Report of the National Cholesterol Education Program (NCEP) Expert Panel on Detection, Evaluation, and Treatment of High Blood Cholesterol in Adults. The Journal of the American Medical Association, 285(19), 2486-2497. 


\section{MHSalud}

URL $\underline{\text { www.una.ac.cr/mhsalud }}$

Bakris, G.L. (2007). Current Perspectives on Hypertension and Metabolic Syndrome. Journal of Managed Care Pharmacy, 13(5): S3-S5

Betteridge, J. (2005). Benefits of lipid-lowering therapy in patients with type 2 diabetes mellitus. The Americam Journal of Medicine, 118(12A), 10S-15S.

Collins, R., Armitage, J., Parish, S., Sleight, P. y Peto, R. (2002). Heart Protection Study of cholesterol lowering with simvastatin in 20,536 high-risk individuals: a randomised placebo-controlled trial. Lancet, 360(9326):7-22.

Egret ${ }^{\circledR}$ for Windows®. Versión 2.0.31. Cytel Software Corporation. 1999

Freeman, D.H. (1987). Applied categorical data analysis. New York: Marcel Dekker Inc.

Grundy, S.M., Becker. D., Clark, L., Cooper, R.S., Denke, M.A., Howard, W.J., et al. (2002). National cholesterol education program expert panel on detection, evaluation, and treatment of high blood cholesterol in adults (Adult Treatment Panel III). Circulation, 106:3143-3421.

Hallin, R., Koivisto-Hursti, U.K., Lindberg, E. y Janson, C. (2006) Nutritional status, dietary energy intake and the risk of exacerbations in patients with chronic obstructive pulmonary disease (COPD). Respiratory Medicine, 100, 561-567. doi:10.1016/j.rmed.2005.05.020

Harrell, F., Lee, K.L., Matchar, D.B. y Reichert, T.A. (1985). Regression models for prognostic prediction: Advantages, problems and suggested solutions. Cancer Treatment Reports, 69(10), 1071-1077.

Jafar, T.H., Qadri, Z. y Chaturvedi, N. (2008). Coronary artery disease epidemic in Pakistan more electrocardiographic evidence of ischemia in women than in men. Heart, 94(4), 408-413.

Kathiresan, S., Manning, A.K., Demissie, S., D'Agostino, R., Surti, A., Guiducci, C.,...Cupples, L. (2007). A genome-wide association study for blood lipid phenotypes in the Framingham Heart Study. BMC Medical Genetics, 8(1), S17. doi:10.1186/1471-2350-8S1-S17.

Klein, S., Burke, L.E., Bray, G.A., Blair, S., Allison, D.B., Pi-Sunyer, X.,... American Heart Association Council on Nutrition, Physical Activity, and Metabolism. (2004). Clinical Implications of Obesity with Specific Focus on Cardiovascular Disease: A Statement for Professionals from the American Heart Association Council on Nutrition, Physical Activity, and Metabolism: Endorsed by the American College of Cardiology Foundation. Circulation, 110(18), 2952-2967.

Kleinbaum, D.G. (1994). Logistic Regression. United States of America: Springer-Verlag New York, Inc. 


\section{MHSalud}

URL $\underline{\text { www.una.ac.cr/mhsalud }}$

Koloszár, S., Fejes, I., Závaczki, Z., Daru, J., Szöllosi, J. y Pál, A. (2005). Effect of body weight on sperm concentration in normozoospermic males. Archives of Andrology, 51(4), 299. 304.

Law, M.R., Wald, N.J., y Thompson, S.G. (1994). By how much and how quickly does reduction in serum cholesterol concentration lower risk of ischaemic heart disease? BMJ, 308:367372.

Mantakas, A. y Farrell, T. (2010). The influence of increasing BMI in nulliparous women on pregnancy outcome. European Journal of Obstetrics, Gynecology, and Reprodutive Biology, 153(1), 43-46.

Meigs, J.B., Wilson, P., Fox, C.S., Vasan, R.S., Nathan, D.M., Sullivan, L.M. \& D’Agostino, R.B. (2006). Body Mass Index, Metabolic Syndrome, and Risk of Type 2 Diabetes or Cardiovascular Disease. The Journal of Clinical Endocrinology \& Metabolism, 91(8), 2906-2912.

Organización Panamericana de la Salud. (2005). Situación de salud en las Américas. Indicadores básicos. Fuente: http://new.paho.org/hq/dmdocuments/2009/IB_SPA_2009.pdf

Perduzzi, P., Concato, J., Kemper, E., Holford, T.R. \& Feinstein, A.R. (1996). A simulation study of the number of events per variable in logistic regression analysis. Journal of Clinical Epidemiology, 49(12), 1373-1379.

Pilote, L., Dasgupta, K., Guru, V., Humphries, K.H., McGrath, J., Norris, C.,...Tagalakis, V. (2007). A comprehensive view of sex-specific issues related to cardiovascular disease. Canadian Medical Association Journal, 176(6), S1 - S44.

Poirier, P., Giles, T.D., Bray, G.A., Hong, Y., Stern, J.S., Pi-Sunyer, F.X. \& Eckel, R.H. (2006). Obesity and Cardiovascular Disease Pathophysiology, Evaluation, and Effect of Weight Loss. Arteriosclerosis, Thrombosis, and Vascular Biology, 26(5), 968-976.

Renault, K., Nørgaard, K., Andreasen, K.R., Secher, N.J. y Nilas, L. (2010). Physical activity during pregnancy in obese and normal-weight women as assessed by pedometer. Acta Obstetrica et Gynecologica Scandinavica, 89(7), 956-961.

Resnick, H.E., Harris, M.I., Brock, D.B. \& Harris, T.B. (2000) American Diabetes Association diabetes diagnostic criteria, advancing age, and cardiovascular disease risk profiles: results from the Third National Health and Nutrition Examination Survey. Diabetes Care, 23(2), 176-180. doi: 10.2337/diacare.23.2.176

Rothman, K.J. (1986). Moderm epidemiology. United States of America: Little, Brown \& Co.

Shepherd, J., Blauw, G.J., Murphy, M.B., Bollen, E., Buckley, B.M., Cobbe, S.M.... Westendorp R.G. (2002). Pravastatin in elderly individuals at risk of vascular disease (PROSPER): a randomized controlled trial. Lancet, 360(9346), 1623-1630.

Tacsan, L. y Asencio, M. (2003). Encuesta basal de factores de riesgo para enfermedades no transmisibles, Cartago 2001. Ministerio de Salud Pública de Costa Rica. 


\section{MHSalud}

URL www.una.ac.cr/mhsalud

Ueshima, H., Kasagi, F., Kodama, K., Okamura, T., Hayakawa, T., Okayama, A... The Nippon Data 80 Research Group. (2006). Risk assessment chart for death from cardiovascular disease based on a 19-year follow-up study of a Japanese representative population. Circulation Journal, 70(10), 1249-1255.

United States Department of Health and Human Services (2004). Seventh Report of the Joint National Committee on Prevention, Detection, Evaluation, and Treatment of High Blood Pressure (JNC 7). Rockville, Md. Publication No. 04-5230.

Watkins, P. (2003). ABC of diabetes, cardiovascular disease, hypertension, and lipids. BMJ, 326, 874-876. doi: $10.1136 / \mathrm{bmj} .326 .7394 .874$

World Health Organization (1995). Physical status: the use and interpretation of anthropometry. Report of a WHO Expert Committee. World Health Organization Technical Report Series. 854, 1-452.

Fecha de recepción: 26 de agosto del 2010.

Fecha de aceptación: 17 de febrero del 2011.

Fecha de publicación: 31 de julio del 2011. 